\title{
Reactions of Microsolvated Organic Compounds at Ambient Surfaces: Droplet Velocity, Charge State, and Solvent Effects
}

\author{
Abraham K. Badu-Tawiah, Dahlia I. Campbell, R. Graham Cooks \\ Department of Chemistry, Purdue University, West Lafayette, IN 47907, USA
}

\begin{abstract}
The exposure of charged microdroplets containing organic ions to solid-phase reagents at ambient surfaces results in heterogeneous ion/surface reactions. The electrosprayed droplets were driven pneumatically in ambient air and then electrically directed onto a surface coated with reagent. Using this reactive soft landing approach, acid-catalyzed Girard condensation was achieved at an ambient surface by directing droplets containing Girard T ions onto a dry ketosteroid. The charged droplet/surface reaction was much more efficient than the corresponding bulk solution-phase reaction performed on the same scale. The increase in product yield is ascribed to solvent evaporation, which causes moderate $\mathrm{pH}$ values in the starting droplet to reach extreme values and increases reagent concentrations. Comparisons are made with an experiment in which the droplets were pneumatically accelerated onto the ambient surface (reactive desorption electrospray ionization, DESI). The same reaction products were observed but differences in spatial distribution were seen associated with the "splash" of the high velocity DESI droplets. In a third type of experiment, the reactions of charged droplets with vapor phase reagents were examined by allowing electrosprayed droplets containing a reagent to intercept the headspace vapor of an analyte. Deposition onto a collector surface and mass analysis showed that samples in the vapor phase were captured by the electrospray droplets, and that instantaneous derivatization of the captured sample is possible in the open air. The systems examined under this condition included the derivatization of cortisone vapor with Girard T and that of 4-phenylpyridine $\mathrm{N}$-oxide and 2-phenylacetophenone vapors with ethanolamine.
\end{abstract}

Key words: Microsolvated compounds, lon/surface reactions, Solvent effects, Mass spectrometry, Charged droplets, Desorption electrospray ionization, lon soft landing

\section{Introduction}

$\mathrm{M}$ icrosolvated compounds represent an important class of chemical entities in nature; they exist in the earth's polar stratosphere as well as in many biological systems. Stratospheric cloud surfaces [nitric acid trihydrate (type I)

Electronic supplementary material The online version of this article (doi:10.1007/s13361-012-0365-3) contains supplementary material, which is available to authorized users.

Correspondence to: R. G. Cooks; e-mail: cooks@purdue.edu and water-ice (type II)] are considered important reaction sites in atmospheric science [1, 2], and the possible significance of charged reagents in such reactions as atmospheric condensation and ice nucleation have been discussed [3]. For example, formation of nitric acid from $\mathrm{N}_{2} \mathrm{O}_{5}$ on ice surfaces is thought to involve microsolvated species [4-8], which include protonated water clusters [5, 6]. In biochemistry, hydrophobic pockets of proteins are known to contain fewer solvent molecules than the rest of the bulk protein. Transport of ions through membranes mediated by proteins [9] also requires that the ion shed some of its solvent molecules [10]; the type of ion $\left(\mathrm{Na}^{+}\right.$or $\left.\mathrm{K}^{+}\right)$transported through 
a particular ion channel thus depends strongly on the hydration energy of the ion [11]. Unfortunately, studies involving microsolvated organic molecules are limited [12, 13], especially as reagents in chemical reactions under atmospheric pressure conditions. Microsolvated peptide ions have been studied in vacuum by Williams and coworkers [14, 15], where the effects of solvation on reactivity are clearly linked to changes in thermochemical properties. Herein, we investigate the reactivity of simple organic ions at ambient surfaces in the presence of small quantities of solvent. The selected reactions have characteristic pathways under bulk solution-phase conditions, and the general goal of this study was to examine the reactivity - including possible new pathways - of the microsolvated species in the open air, and to interpret the results in the light of solvent effects.

Experimental [16-19] and theoretical [20] studies on gasphase ion/molecule reactions of microsolvated ions have included the investigation of solvent effects [21, 22]. The experimentally observed trend is a reduction in the reaction efficiency, which starts with monosolvation. Searles and Kebarle made some of the first detailed observations of the effects of solvation on alkali cations (M), and observed an increase in the enthalpy of formation of hydrated species in the series $\mathrm{M}\left(\mathrm{H}_{2} \mathrm{O}\right)_{n}{ }^{+}$where $n$ ranges from $n=1$ upwards [23, 24]. Theoretical investigations reveal a systematic transformation of the gas-phase double-well potential to an energy profile for nucleophilic substitution with much shallower reactant and product complex minima and an increased activation barrier upon successive hydration [16, 25, 26]. Thus, the energy profiles of gas-phase microsolvated ion/ molecule reactions approach those for bulk solution-phase reactions (except for a few instances [12]), in which large activation energies are typically required.

Interestingly, increased reaction rates have been observed for certain solution-phase reactions conducted under microreactor conditions, in which the presence of microsolvated species cannot be ruled out. Such microreactor systems have larger surface areas, which affect physical processes, such as evaporation, and support partitioning of solutes from the droplet bulk to droplet surface [27-29]. Examples of such microreactor systems include the creation of gas/vapor-phase bubbles in liquids by ultrasonication $[30,31]$ as well as the recent observations of accelerated reactions in charged microdroplets by reactive desorption electrospray ionization (reactive DESI [32]). Aside from the accelerated reaction rates, the DESI methodology has also been shown to be efficient in capturing transient reaction intermediates in bulk solution-phase on millisecond time scale [33]. In sonochemistry, acoustic cavitation causes the formation, growth, and implosive collapse of bubbles in a liquid, releasing large amounts of heat $\left(5000{ }^{\circ} \mathrm{C}\right.$ within lifetimes of a few microseconds) that increase rates of chemical reaction by several orders of magnitude. In reactive DESI experiments by contrast, smaller droplets generated upon evaporative cooling of solution-phase charged microdroplets are thought to increase the rates of acid/base catalyzed solution-phase bimolecular reactions [32]. To the best of our knowledge, heterogeneous surface reactions utilizing charged microdroplets have not been reported, although heterogeneous gas-phase ion/surface reactions under vacuum are common $[34,35]$.

The instrumentation used for the vacuum-base ion/surface reactions is based on the ion soft landing (SL) technique [36-38] in which mass-selected polyatomic ions are deposited intact onto a surface using a mass spectrometer. For surface reactions, the selected ion is given enough energy to react selectively with particular functional groups present at the surface. Recently, the SL technique was extended from vacuum to ambient conditions in a process termed ambient soft landing [39]. In ambient ion soft landing, polyatomic organic ions are deposited from air onto a surface at a specified location under atmospheric pressure conditions. The present work utilized the ambient soft landing apparatus except that charged microdroplets, instead of dry ions, were selected for deposition and reaction at an ambient surface.

Three different experimental conditions are utilized in this study on the Girard condensation as the test reaction: (1) ambient reactive soft landing utilizing slow velocity droplets, (2) reactive DESI in which high velocity droplets are used [40], and (3) vapor phase analyte capture and reaction in charged microdroplets. The charged droplets used in this study were generated using electrosonic spray ionization (ESSI [41]) because it allows transport of the electrosprayed droplets over a greater distance. The advantage of this type of electrospray (ESI [29, 42, 43]) is that it allows some solvent evaporation from the droplets during their transfer to the designated location, and the use of the derived charged microdroplets for surface reactions at atmospheric pressure. To effect surface reaction, the charged microdroplets containing the desired reagent ion were directed onto the reaction surface containing a nominally dry reagent using the ambient soft landing apparatus [39, 44]. Under particular experimental conditions (discussed later), this apparatus allows focusing of the ions onto a $2 \mathrm{~mm}$ (diameter) spot size, producing no observable macroscopic solvent on the surface. Ion/surface reactions were achieved upon interaction of the microdroplets with the reagent already present at the surface. Results indicated increased product yields for the surface reaction compared with the corresponding bulk solution-phase reactions, and possible factors influencing the observed rate enhancement are discussed. The vaporphase analyte capture experiment was achieved by modifying the ambient soft landing apparatus to allow charged droplets doped with a reagent to intercept the headspace vapor of an analyte (after being heated) in an enclosed chamber. Vapor-phase analytes, which are captured by the charged droplets, are then directed onto a surface for collection and characterization. Removal of the deposited material and analysis by MS showed that reaction of the captured analyte with reagents contained in charged droplets is possible in the open laboratory [45-48]. As will be shown, the charged droplet environment (either at a surface or in air) 
provides the required conditions in which solvated organic ions can be utilized effectively for acid catalyzed reactions.

\section{Experimental}

\section{Instrumentation}

The main components of the simple apparatus used for the generation and deposition of microdroplets consisted of an ESSI ion source and ion deflecting and collecting electrodes (Figure 1). A straight metal tube was inserted between the ion source and the deflecting electrodes to collimate the droplets entering the deflector electrode. The distance between the ESSI ion source silicon capillary tip and the metal tube was kept at $1 \mathrm{~mm}$ to minimize sample loss due to expansion of the electrospray plume. Reaction at the collector surface was achieved by electrically directing the charged microdroplets doped with reagent onto a surface set orthogonal to the flow of the droplets, and on which dry analyte had been coated. This surface was usually biased with an appropriate voltage, for example $-6 \mathrm{kV}$ for positive ion deposition. The other two electrodes are grounded, as shown in Figure 1a. The middle electrode is a $5 \mathrm{~mm}$ thick $(5 \times 5 \mathrm{~cm}$ square $)$ stainless steel with a circular hole $(1 \mathrm{~cm}$ in diameter) at its center. It allows ions to be focused onto the collector/reaction surface [39]. The typical diameter of the ion spot observed under this condition was $2 \mathrm{~mm}$, but actual spot size depended not only on the thickness of the middle electrode but also on the magnitude of the DC potentials applied [39]. Sample solutions of 50-2000 ppm concentration were prepared in methanol or acetonitrile. The sample solutions were electrosprayed using a spray voltage of $\pm 7 \mathrm{kV}$, nebulizer gas $\left(\mathrm{N}_{2}\right)$ pressure of $120 \mathrm{psi}$, and sample injection rate of $5 \mu \mathrm{L} / \mathrm{min}$. The $\mathrm{pH}$ of the landing positively and negatively charged microdroplets was measured using ultra-sensitive $\mathrm{pH}$ paper (pHydrionUltrafine; Micro Essential Laboratory, Brooklyn, NY, USA) placed at the landing site.

\section{Nanospray Ionization Mass Spectrometry for Product Analysis}

After surface reaction, the spots on which ions had impinged were washed with $10 \mu \mathrm{L}$ of methanol/water $(1: 1, \mathrm{vol} / \mathrm{vol})$ and characterized using nanospray-MS. Nanospray $(\sim 20 \mathrm{~nL} /$

(a)

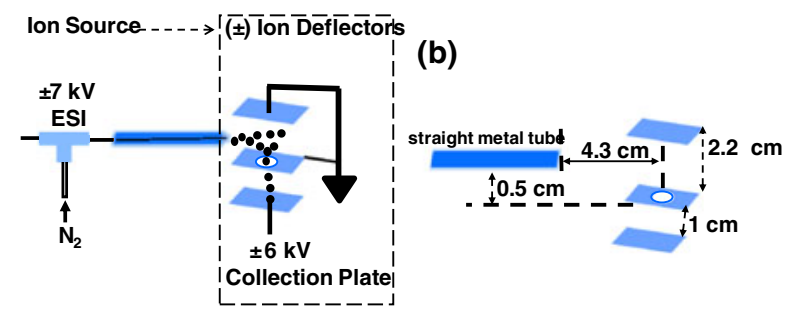

Figure 1. (a) Experimental apparatus for ambient surface reactions showing applied potentials and charged droplet deflection, (b) dimensions; straight metal tube: length $7.5 \mathrm{~cm}$, $0.5 \mathrm{~cm}$ i.d., $0.6 \mathrm{~cm}$ o.d min flow rate) voltage of $1.8 \mathrm{kV}$ was supplied to the emitter. These sample analysis conditions involve small droplets and short ionization periods; no significant reaction is observed during analysis as shown by the control experiments. All MS experiments were performed using a Thermo Fisher Scientific LTQ mass spectrometer (San Jose, CA, USA). Typical MS parameters used included averaging of 3 microscans, $100 \mathrm{~ms}$ maximum ion injection time, $15 \mathrm{~V}$ capillary voltages, $150{ }^{\circ} \mathrm{C}$ capillary temperature, and $65 \mathrm{~V}$ tube lens voltage. Data were acquired and processed using Xcalibur 2.0 software (Thermo Fisher Scientific). The identification of analyte ions was confirmed by tandem mass spectrometry using collision-induced dissociation (CID). An isolation window of $1.5 \mathrm{Th}$ (mass/charge units) and a normalized collision energy of $30 \%-35 \%$ (manufacturer's unit) was selected for the CID experiment.

\section{Chemicals and Reagents}

Cortisone, androsterone, testosterone, Girard $\mathrm{T}$ reagent, and 4-phenylpyridine $N$-oxide were purchased from SigmaAldrich (Milwaukee, WI, USA), whereas ethanolamine was obtained from Acros Organics (Geel, Belgium), and methanol and acetonitrile (HPLC grade) from Mallinckrodt Baker Inc. (Phillipsburg, NJ, USA). Deionized/distilled water was obtained using a Barnstead/Thermolyne deionizer unit (Barnstead Mega-Pure System, Dubuque, IA, USA).

\section{Results and Discussion}

\section{Charged Droplet/Surface Reactions}

Aldehydes and ketones react slowly with Girard reagents in the presence of acid to form the corresponding hydrazones [49]. In this study, Girard T (GT) reaction with keto-steroids was achieved at ambient surfaces by allowing charged microdroplets containing GT to impinge on the dry steroid. A nanospray mass spectrum of the removed surface reaction product and, for purpose of comparison, a nanospray-MS of the bulk solution-phase product of reaction of GT and cortisone are shown in Figure 2. The data demonstrate that the reaction occurred on the surface before the washing needed for nESI mass spectrometry (MS) analysis and that the reaction yield is enhanced by the use of charged microdroplets compared with bulk solution-phase conditions (Figure 2). Similar effects were recorded for testosterone and androsterone (Figures S1 and S2, Supporting Information). In all cases, the Girard condensation product underwent a characteristic trimethylamine neutral loss under collisioninduced dissociation (CID) experiments to yield ions at $\mathrm{m} / \mathrm{z}$ 415,345 , and 343 for cortisone, androsterone, and testosterone, respectively. Under the same charged droplet/surface experimental conditions, more reaction product was collected for the GT reaction with cortisone compared with that collected from testosterone, which in turn yielded more product than did the sterically hindered androsterone. 

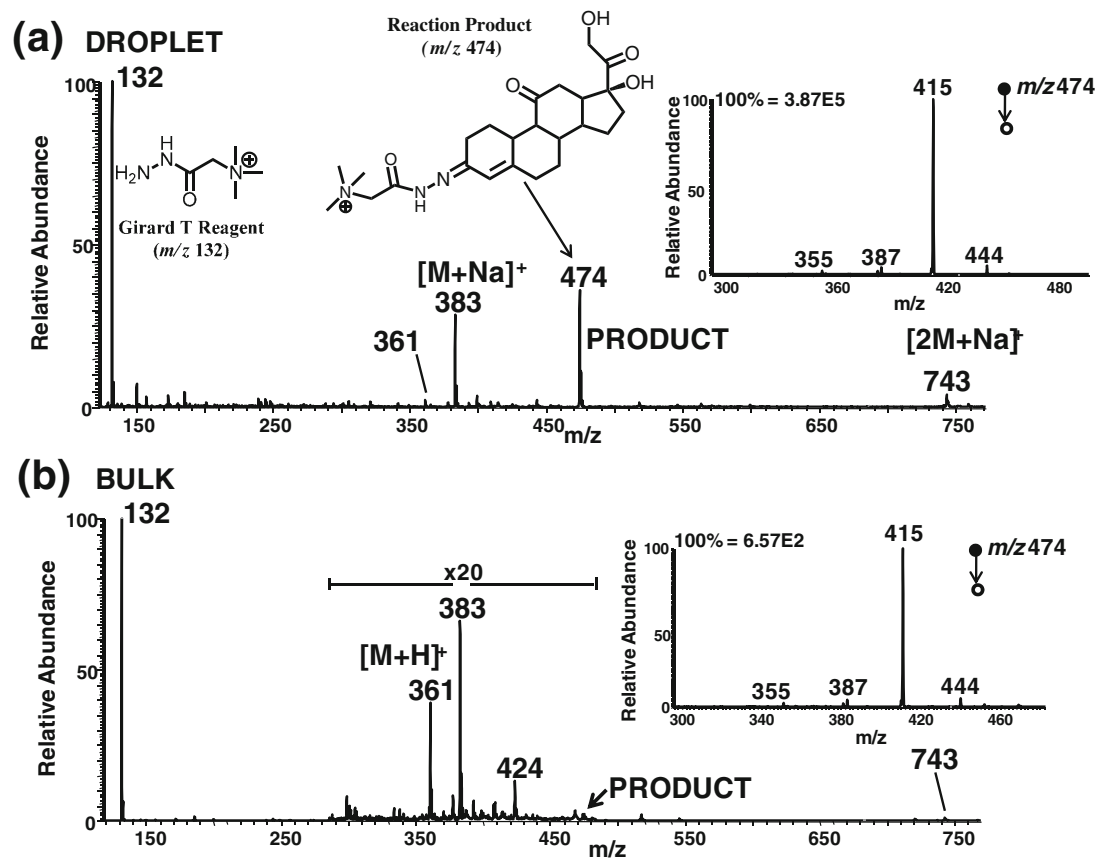

Figure 2. Nanospray-MS of Girard T and cortisone (M) reaction mixture after 30 min of reaction time: (a) at ambient surface and (b) in bulk solution-phase. Girard T (GT) in acetonitrile solution (50 ppm) was sprayed as $5 \mu \mathrm{L} / \mathrm{min}$ in (a), whereas $7.5 \mu \mathrm{L}$ (corresponding to amount of GT directed at the surface in $30 \mathrm{~min}$.) of the same solution was utilized for the solution-phase reaction. The same amounts of cortisone ( $2 \mu \mathrm{L}$ of $100 \mathrm{ppm}$ solution in acetonitrile) were used in both (a) and (b). Solution-phase reaction mixture was allowed to stand at ambient temperature for $30 \mathrm{~min}$, after which $10 \mu \mathrm{L}$ of methanol/water (1:1, vol/vol) was added for MS analysis. Similarly, the surface reaction was washed using $10 \mu \mathrm{L}$ of methanol/water (1:1, vol/vol) after microdroplets containing GT had impinged on the dried cortisone for $30 \mathrm{~min}$. Inserts indicate MS/MS product ion spectrum of reaction product at $m / z 474$

As mentioned in the Introduction, increased reaction rates relative to bulk solution have previously been observed for the GT/cortisone reaction system under DESI conditions [32]. In the reactive DESI experiment, high velocity $(100 \mathrm{~m} / \mathrm{s})$ electrospray solvent droplets containing dissolved reagent arriving at a surface form a localized liquid thin film, which dissolves analyte present on the surface. Subsequent droplet/liquid-thinfilm collisions produce secondary microdroplets containing both the reagent and the analyte, which are then sucked into the mass spectrometer for mass/charge analysis. Chemical reactions are observed to occur within the DESI analysis time scale (milliseconds), inside the departing secondary droplets when reactive reagents including Girard $\mathrm{T}$ are added to the DESI spray solvent $[50,51]$. The rate enhancement associated with the reactive DESI process was attributed to solvent evaporation leading to extreme $\mathrm{pH}$ changes and reagent concentration. The reactive soft landing method differs from the reactive DESI experiment in being a heterogeneous ion/surface reaction as opposed to the homogeneous droplet reaction in the case of DESI. No splashes were observed during microdroplet deposition (Figure 3c and e) consistent with the small number of solvent molecules, and the low velocity of the landing droplets. The impacting DESI primary droplets move rapidly and splash product across the surface. The spatial profile of the products of these experiments is consistent with this interpretation (Figure 3).
Two main factors have been identified as influencing the increased reaction rate observed for the GT reaction with the ketones at the surface, the effects of charge and solvent. Charge effects were investigated by supplying $+7,-7$, and $0 \mathrm{kV}$ voltages independently to the GT spray solution and directing the derived electrosprayed droplets onto the reaction surface containing dried cortisone. The results indicated that more reaction product $(\mathrm{m} / \mathrm{z} 474)$ was collected for $+7 \mathrm{kV}$ spray voltage, followed by $-7 \mathrm{kV}$ (Figure S3, Supporting Information). This result is not surprising because the GT reaction with ketone is acid catalyzed and, in fact, the $\mathrm{pH}$ of the landing positively charged microdroplets (after traveling a distance of $12 \mathrm{~cm}$ ) generated by the $+7 \mathrm{kV}$ spray voltage was measured to be 1.5 , whereas the $\mathrm{pH}$ of landing negatively charged droplets (generated using $-7 \mathrm{kV}$ spray voltage) was found to be 2.5 . The poor performance observed when no voltage was supplied to the spray solution is attributed to fewer GT reagents at the reaction surface and insufficient $\mathrm{pH}$ modification due to the lack of charge in the landing microdroplets $(\mathrm{pH}$ could not be measured possibly because the droplets are too small). The $\mathrm{pH}$ of the droplets immediately after exiting ESI source was measured to be 3.5 (positive droplets) and 4.0 (negative droplets). No product ion $(\mathrm{m} / \mathrm{z} 474)$ was observed when the GT was prepared in a solution of $\mathrm{pH} 10$. The $\mathrm{pH}$ of the landing droplets was 12.5 in this case for negatively charged 


\section{Ambient Reactive Soft Landing}

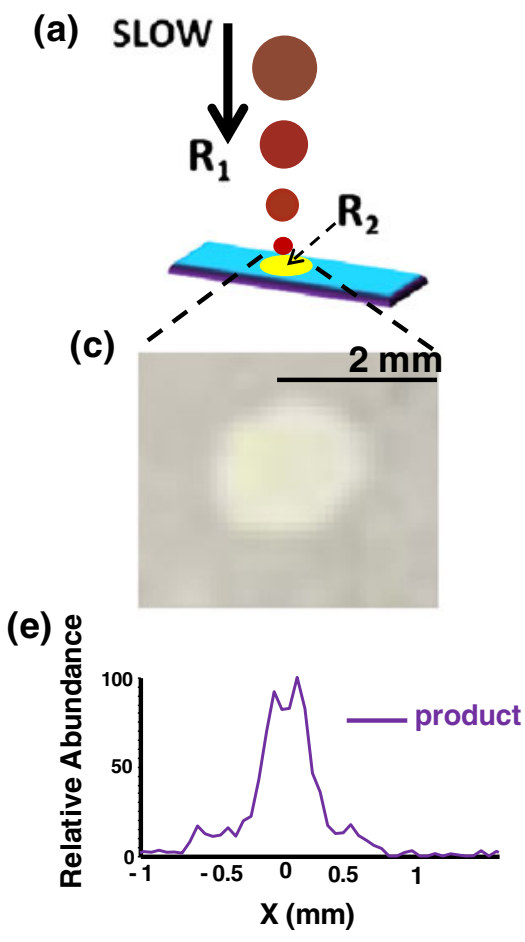

\section{Ambient Reactive DESI}

(b)

(d)

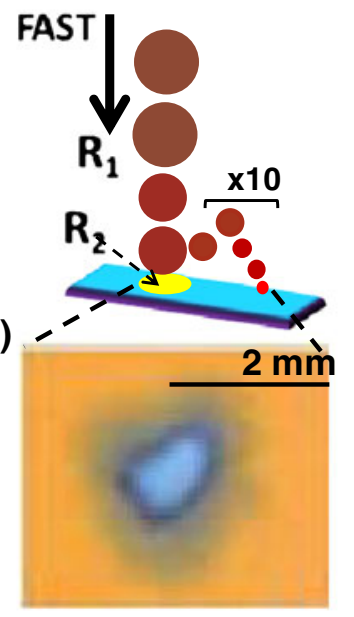

(f)

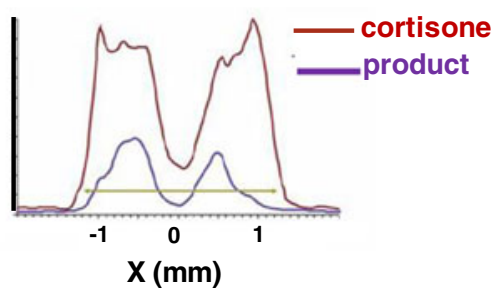

Figure 3. Contrasting reaction product distributions created upon impact of electrospray droplets using (a) ambient soft landing apparatus, utilizing slow velocity droplets and (b) desorption electrospray ionization (DESI) using high velocity droplets. Soft landing yields product spot in which product ions $(\mathrm{m} / \mathrm{z} 474)$ are concentrated at its center (c) and (e), whereas in the DESI experiment products are depleted from the center of the impact point (d) and (f). Conditions of DESI analysis of ion spot in (c): $\mathrm{MeOH} / \mathrm{H}_{2} \mathrm{O}(1: 1, \mathrm{vol} / \mathrm{vol})$ as the spray solvent, $50^{\circ}$ incident angle to the horizontal, $3 \mu \mathrm{L} / \mathrm{min}$ solvent flow rate, 150 psi gas (N2) pressure, $+4.5 \mathrm{kV}$ spray voltage, $175 \mu \mathrm{m} / \mathrm{s}$ moving stage velocity. Parts (d) and (f) are reproduced with permission from reference [32] for comparison with reactive soft landing

droplets. Aside from charge polarity (i.e., positive versus negative droplets), the type of ions (protonated versus sodiated, etc.) contained inside the charged microdroplets was also found to affect reaction efficiency. For example, a large reduction in reaction yield was observed when the keto-steroid (e.g., androsterone) solution in acetonitrile was electrosprayed, and the resulting positively charged microdroplets were allowed to interact with dried GT present at the surface (Figure S4, Supporting Information). In this reverse experiment (spraying the keto-steroid instead of GT), the charged droplets were determined to contain sodiated/ potassiated dimers and trimers of the keto-steroid, with no or little protonated monomeric species. Since the GT reaction with ketone is acid catalyzed, the protonated ketone can be considered more reactive than the sodiated or potassiated species (Figure S5, Supporting Information), and so the absence of such protonated species should ultimately result in reduction in the reaction efficiency.

It seems likely that solvent evaporation from the microdroplets will ultimately lead to microsolvation of reagents during (1) transfer to the reaction surface, and/or (2) during residence time on the surface. Thus, the effect of solvent evaporation on reaction rate was investigated by performing a delayed dissolution experiment using the Girard $\mathrm{T}$ and cortisone reaction system. That is, following reagent deposition, the reaction mixture was either kept in the open air or under a dry environment inside a dessiccator, for a given time before washing. The open-air condition showed a modest 2-fold increase in product yield as a function of delay time (time over which the reaction mixture was kept before dissolving it into solution for MS analysis), within a 30 min delay time (Figure S6a, Supporting Information). In the dry environment, however, no significant increase in reaction yield was observed irrespective of the delay time. These results indicate that evaporation of the microscopic amounts of solvent present at the surface led to a significant increase in reaction yield, even after droplet deposition had ceased. This effect was further investigated simply by pipetting $2 \mu \mathrm{L}$ each of the GT (50 ppm) and cortisone $(100 \mathrm{ppm})$ solutions in acetonitrile onto a surface in the open air. The resulting dried reaction mixture was dissolved in $10 \mu \mathrm{L}$ of methanol/water $(1: 1, \mathrm{vol} / \mathrm{vol})$ solution and analyzed using nanospray-MS. The results of this experiment also showed that the longer the reaction mixture was 


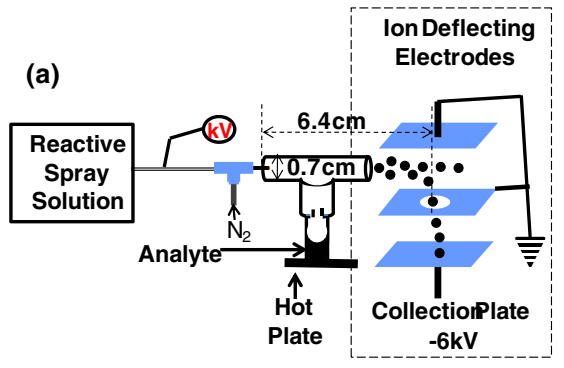

(b)

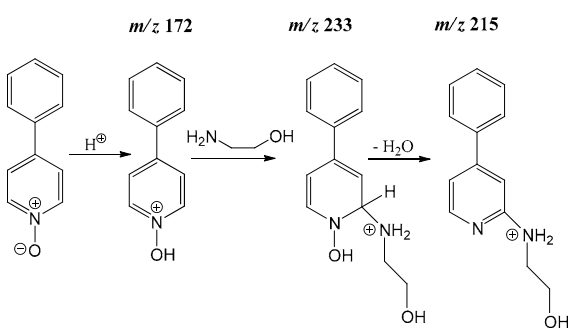

Figure 4. (a) Apparatus for droplet-vapor reaction, (b) reaction of charged microdroplets containing ethanolamine with 4-phenylpyridine $\mathrm{N}$-oxide vapor

allowed to stand in the open air, the more product was collected (red trace, Figure S6b, Supporting Information), hence confirming the effect of evaporation on reaction yield. Again, due to the combined effects of charge and solvent evaporation, more products were collected for charged microdroplets than from the neutral droplets derived from drop-casting.

\section{Charged Droplet/Vapor Capture and Reaction}

Charged microdroplet reaction with vapor samples in the open air was also achieved using a modified apparatus
(Figure 4a). Here, a reagent was added to the spray solvent, and the electrosprayed droplets containing the reagent were allowed to intercept the vapor of an analyte in an enclosed chamber (i.d. $0.7 \mathrm{~cm}$, length $3.5 \mathrm{~cm}$ ). The vapor-phase analyte was captured by the charged droplets, and then the droplet and its contents were directed onto the collector surface. Dissolution of the landed material was achieved using $10 \mu \mathrm{L}$ of methanol/water $(1: 1, \mathrm{vol} / \mathrm{vol})$ solution, which was followed by nESI-MS analysis. For example, the results of the mass analysis of the collected material after exposing 4-phenylpyridine $\mathrm{N}$-oxide vapor to ethanolamine dissolved
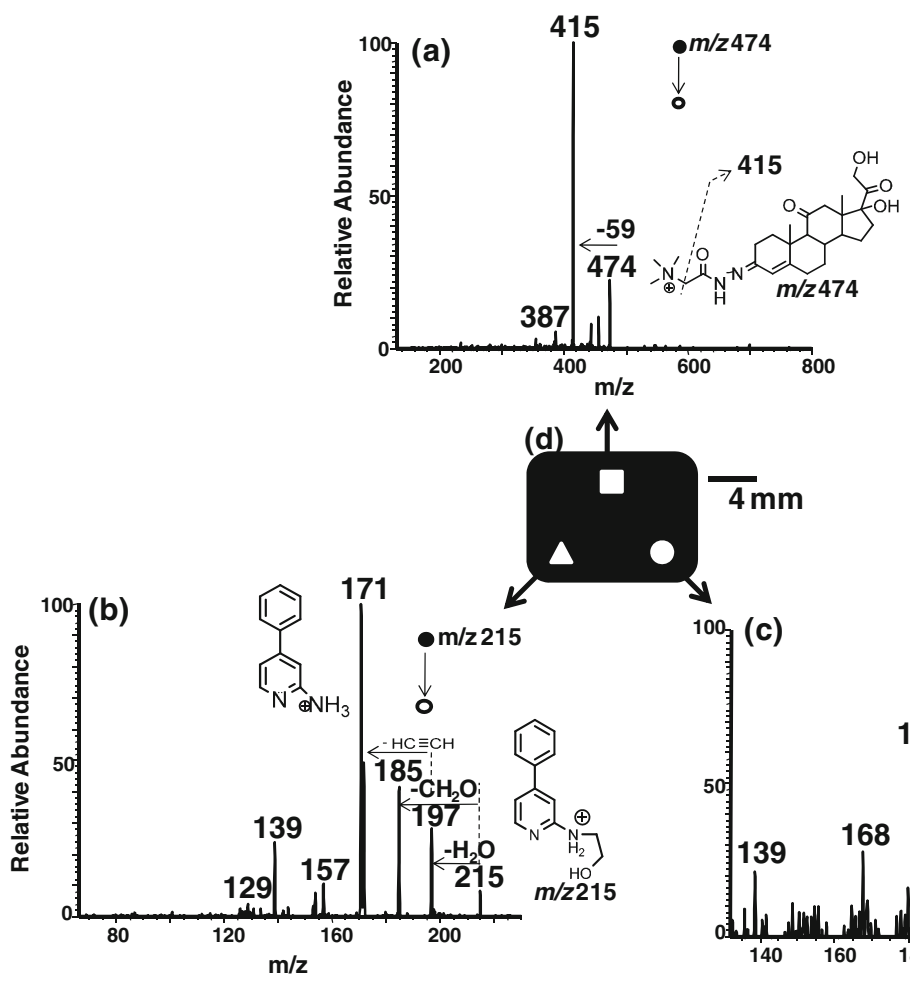

个 
in charged microdroplets (Figure 4b) is shown in Figure 5b. These MS/MS spectra recorded for the collected ions (also see Figure S7, Supporting Information for full mass spectra) show that charged microdroplets can capture the vapor of an analyte as already reported by others [47, 48], and also that in the presence of a reagent the charged microdroplets become reaction vessels where chemical reactions occur. In other words, online derivatization of chemical species during transfer to the landing surface is possible. This was examined further by instantaneously derivatizing and depositing the products generated from three different chemical species separately at different positions on the same surface as depicted at the center of Figure 5 (i.e., Figure $5 \mathrm{~d}$ ). The three reactions shown are the derivatization of cortisone with Girard $\mathrm{T}$ reagent (Figure 5a) 4-phenylpyridine $N$-oxide (Figure $5 \mathrm{~b}$ ) with ethanolamine and 2-phenylacetophenone (Figure 5c) with ethanolamine. Note also that reaction is expected to continue during the time of droplet residence on the surface.

\section{Conclusion}

The reactivity of organic ions has been studied at atmospheric pressure under two different experimental conditions: (1) using reactive soft landing and (2) using vaporphase capture by charged droplets, and comparisons have been made with a third experiment (3) reactive DESI. The reactions of interest include cortisone reaction with Girard T reagent and ethanolamine reaction with 4-phenylpyridine $\mathrm{N}$ oxide and with 2-phenylacetophenone. More than an order of magnitude increase in product yield was observed for the charged droplet/surface reaction compared with the corresponding bulk solution-phase reactions performed using the same amounts of material. Electrospray appears to provide an efficient way to generate highly reactive microsolvated organic ions in the form of charged microdroplets in ambient air. Ambient ion soft landing allows ions and charged droplets to be effectively transported, focused, and deposited gently at a designated location for reaction with a chosen reagent. While most reactive species are expected to be the fully desolvated ions, these might be more difficult to deal with in quantity, so the conditions and rate enhancements seen here are associated with partial (and poorly characterized) degrees of desolvation. Unlike ambient reactive DESI experiments in which rate enhancements are also seen, the procedures used in this work do not involve fast-moving droplets so they do not generate splashes upon surface impact of the primary droplets. Reactions are limited to those that occur at the impact site. The observed rate enhancements associated with this reactive soft landing are ascribed to both charge and solvent effects. The use of charged droplets allows extremes of $\mathrm{pH}$ to be reached in the droplet arriving at the impact point while solvent evaporation also provides increased reagent concentration. Both factors allow the reaction to approach those used in isolatedphase ion/molecule reactions, which often occur upon every collision.
Charged microdroplets also served as reaction vessels to capture gas-phase analytes - a known process [45] — and to follow this with previously unknown reaction. This sequence enabled direct capture of gas-phase analyte in ambient air, followed by their reaction and then transfer of the derivatized product onto a surface for collection and independent characterization.

\section{Acknowledgment}

The authors acknowledge funding for this work by the National Science Foundation (CHE NSF 0848650).

\section{References}

1. Nielsen, S.B., Andersen, L.H.: Properties of microsolvated ions: from the microenvironment of chromophore and alkali metal ions in proteins to negative ions in water clusters. Biophys. Chem. 124, 229-237 (2006)

2. MacTaylor, R.S., Castleman Jr., A.W.: Cluster ion reactions: insights into processes of atmospheric significance. J. Atmos. Chem. 36, 23-63 (2000)

3. Harrison, R.G.: Cloud formation and the possible significance of charge for atmospheric condensation and ice nuclei. Space Sci. Rev. 94, 381396 (2000)

4. Molina, M.J., Tso, T., Molina, L.T., Wang, F.C.-Y.: Antarctic stratospheric chemistry of chlorine nitrate, hydrogen chloride, and ice: release of active chlorine. Science 238, 1253-1257 (1987)

5. Crutzen, P.J., Arnold, F.: Nitric acid cloud formation in the cold antarctic stratosphere: a major cause for the springtime 'Ozone Hole'. Nature 324, 65 (1986)

6. Bohringer, H., Fahey, D.W., Fehsenfeld, F.C., Ferguson, E.E.: The role of ion-molecule reactions in the conversion of N2O5 to HNO3 in the stratosphere. Planet Space Sci 31, 185-191 (1983)

7. Worsnop, D.R., Zahniser, M.S., Fox, L.E., Wofsey, S.C.: Vapor pressures of solid hydrates of nitric acid: implications for polar stratospheric clouds. Science 259, 71 (1993)

8. Horn, A.B., Chesters, M.A., McCoustra, M.R.S., Sodeau, R.: Adsorption of stratospherically important molecules on thin $\mathrm{D}_{2} \mathrm{O}$ ice films using reflection absorption infrared spectroscopy. J. Chem. Soc. Faraday Trans. 88(7), 1077-1078 (1992)

9. Hille, B.: Ion channels of excitable membranes, 3rd edn. Sinauer Associates, Sunderland (2001)

10. Stryer, L.: Biochemistry, 3rd edn. W.H. Freeman and Company, New York (1988)

11. Gouaux, E., MacKinnon, R.: Principles of selective ion transport in channels and pumps. Science 310, 1461 (2005)

12. Aschi, M., Attina, M., Cacace, F., Ricci, A.: Experimental study on the mechanism of gas-phase aromatic nitration by protonated methyl nitrate. J. Am. Chem. Soc. 116(21), 9535-9542 (1994)

13. Chabinyc, M.L., Craig, S.L., Regan, C.K., Brauman, J.I.: Gas-phase ionic reactions: dynamics and mechanism of nucleophilic displacements. Science 279, 1882-1886 (1998)

14. Schnier, P.D., Gross, D.S., Williams, E.R.: Electrostatic forces and dielectric polarizability of multiply protonated gas-phase cytochrome- $c$ ions probed by ion/molecule chemistry. J. Am. Soc. Mass Spectrom 6, 1086-1097 (1995)

15. Chakraborty, T., Holm, A.I.S., Hvelplund, P., Nielsen, S.B., Poully, J.-C., Worm, E.S., Williams, E.R.: On the survival of peptide cations after electron capture: role of internal hydrogen bonding and microsolvation. $J$. Am. Soc. Mass Spectrom 17, 1675-1680 (2006)

16. O'Hair, R.A.J., Davico, G.E., Hacaloglu, J., Dang, T.T., DePuy, C.H., Bierbanm, V.M.: Measurements of solvent and secondary kinetic isotope effects for the gas-phase $\mathrm{SN} 2$ reactions of fluoride with methyl halides. J. Am. Chem. Soc. 116, 3609-3610 (1994)

17. van der Wel, H., Nibbering, N.M.M., Sheldon, J.C., Hayes, R.N., Bowie, J.H.: Nucleophilic substitution in the gas phase. The reactions of alkoxide-alkanol negative ions with boron, carbon, silicon, and titanium alkoxides. An ion cyclotron resonance and ab initio study. J. Am. Chem. Soc. 109, 5823 (1987) 
18. Bickelhaupt, F.M., Baerends, E.J., Nibbering, N.M.M.: The effect of microsolvation on E2 and SN2 reactions: theoretical study of the model system $\mathrm{F}^{-}+\mathrm{CH} 3 \mathrm{CH} 2 \mathrm{~F}+$ nHF. Chem. Eur. J. 2, 196-207 (1996)

19. Freriks, I.L., de Koning, L.J., Nibbering, N.M.M.: Gas-phase ambient reactivity of monohydrated enolate anions. J. Org. Chem. 57, 59765979 (1992)

20. Ohta, K., Morokuma, K.: An MO study of SN2 reactions in hydrated gas clusters: hydrated hydroxide $\left[\left(\mathrm{H}_{2} \mathrm{O}\right) \mathrm{nOH}-\right]+$ hydrated methyl chloride $[\mathrm{MeCl}(\mathrm{H} 2 \mathrm{O}) \mathrm{m}]$.fwdarw. Methanol + chloride $+(\mathrm{n}+\mathrm{m})$ Water. J. Phys. Chem. 89, 5845 (1985)

21. Bohme, D.K., Raksit, A.B.: Gas-phase measurements of the influence of stepwise solvation on the kinetics of nucleophilic displacement reactions with chloromethane and bromomethane at room temperature. J. Am. Chem. Soc. 106, 3447 (1984)

22. Hierl, P.M., Ahrens, A.F., Henchman, M.J., Viggiano, A.A., Paulson, J. F.: Chemistry as a function of solvation number. Solvated-ion reactions in the gas phase and comparison with solution. Faraday Discuss. Chem. Soc. 85, 37 (1988)

23. Searles, S.K., Kebarle, P.: Hydration of the potassium ion in the gas phase: enthalpies and entropies of hydration reactions $\mathrm{K}^{+}\left(\mathrm{H}_{2} \mathrm{O}\right) \mathrm{n}-1+$ $\mathrm{H}_{2} \mathrm{O}=\mathrm{K}^{+}(\mathrm{H} 2 \mathrm{O}) \mathrm{n}$; for $\mathrm{n}=1$ to $\mathrm{n}=6$. Can. J. Chem. 47, 2619 (1969)

24. Dzidic, I., Kebarle, P.: Hydration of the alkali ions in the gas phase. Enthalpies and entropies of reactions $\mathrm{M}+(\mathrm{H} 2 \mathrm{O}) \mathrm{n}-1+\mathrm{H}_{2} \mathrm{O}=\mathrm{M}+$ $\left(\mathrm{H}_{2} \mathrm{O}\right)$ n. J. Phys. Chem. 74, 1466 (1970)

25. Tucker, S.C., Truhlar, D.G.: A six-body potential energy surface for the $\mathrm{SN} 2$ reaction $\mathrm{Cl}-(\mathrm{g})+\mathrm{CH} 3 \mathrm{Cl}(\mathrm{g})$ and a variational transition-state-theory calculation of the rate constant. J. Am. Chem. Soc. 112(9), 3338-3347 (1990)

26. Craig, S.L., Brauman, J.I.: Intramolecular microsolvation of thermoneutral gas phase SN2 reactions. J. Am. Chem. Soc. 118, 6786-6797 (1996)

27. Jorabchi, K., Smith, L.M.: Single droplet separations and surface partition coefficient measurements using laser ablation mass spectrometry. Anal. Chem. 81(23), 9682-9688 (2009)

28. Ablyazov, P.N., Vasilevskaya, V.V., Khokhlov, A.R.: Reactions in surface microreactors: computer simulation. Colloid J. 69(3), 265-271 (2007)

29. Cech, N.B., Enke, C.G.: Relating electrospray ionization response to nonpolar character of small peptides. Anal. Chem. 72, 2717-2723 (2000)

30. Suslick, K.S.: Sonochemistry. Science 247, 1439-1445 (1990)

31. Kuijpers, M.W.A., van Eck, D., Kemmere, M.F., Keurentjes, J.T.F.: Cavitation-induced reactions in high-pressure carbon dioxide. Science 298, 1969-1971 (2002)

32. Girod, M., Moyano, E., Campbell, D.I., Cooks, R.G.: Accelerated bimolecular reactions in microdroplets studied by desorption electrospray ionization mass spectrometry. Chem. Sci. 2, 501-510 (2011)

33. Perry, R.H., Splendore, M., Chien, A., Davis, N.K., Zare, R.N.: Detecting reaction intermediates in liquids on the millisecond time scale using desorption electrospray ionization. Angew. Chem. Int. Ed. 50, 250-254 (2011)
34. Gologan, B., Green, J.R., Alvarez, J., Laskin, J., Cooks, R.G.: Ion/ surface reactions and ion soft-landing. Phys. Chem. Chem. Phys. 7, 1490-1500 (2005)

35. Hu, Q., Wang, P., Gassman, P.L., Laskin, J.: In situ studies of soft- and reactive landing of mass-selected ions using infrared reflection absorption spectroscopy. Anal. Chem. 81, 7302-7308 (2009)

36. Franchetti, V., Solka, B.H., Baitinger, W.E., Amy, J.W., Cooks, R.G.: Soft landing of ions as a means of surface modification. Int. J. Mass Spectrom. Ion Phys. 23(1), 29-35 (1977)

37. Siuzdak, G., Hollenbeck, T., Bothner, B.: Preparative mass spectrometry with electrospray ionization. J. Mass Spectrom. 34, 1087 (1999)

38. Rauschenbach, S., Stadler, F.L., Lunedei, E., Malinowski, N., Koltsov, S., Costantini, G., Kern, K.: Electrospray ion beam deposition of clusters and biomolecules. Small 2(4), 540-547 (2006)

39. Badu-Tawiah, A.K., Wu, C., Cooks, R.G.: Ambient ion soft landing. Anal. Chem. 83, 2648-2654 (2011)

40. Venter, A., Sojka, P.E., Cooks, R.G.: Droplet dynamics and ionization mechanisms in desorption electrospray ionization mass spectrometry. Anal. Chem. 78(24), 8549-8555 (2006)

41. Takats, Z., Wiseman, J.M., Gologan, B., Cooks, R.G.: Electrosonic spray ionization. A gentle technique for generating folded proteins and protein complexes in the gas phase and studying ion-molecule reactions at atmospheric pressure. Anal. Chem. 76, 4050-4058 (2004)

42. Fenn, J.B., Mann, M., Meng, C.K., Wong, S.F., Whitehouse, C.M.: Electrospray ionization for mass spectrometry of large biomolecules. Science 246(4926), 64-71 (1989)

43. Cole, R.B.: Electrospray Ionization Mass Spectrometry: Fundamentals Instrumentation and Applications, 1st edn. John Wiley and Sons, New York (1997)

44. Badu-Tawiah, A.K., Cyriac, J., Cooks, R.G.: Reactions of organic ions at ambient surfaces in a solvent-free environment. J. Am. Soc. Mass Spectrom. (2012). doi:10.1007/s13361-012-0337-7

45. Fuerstenau, S., Kiselev, P., Fenn, J.B.: ESIMS in the analysis of trace species in gases. Proceedings of the 47th ASMS Conference on Mass Spectrometry Allied Topics, Dallas, TX, June, (1999)

46. Kiselev, P., Fenn, J.B.: ESIMS Analysis of Vapors at Trace Levels. Proceedings of the 49th ASMS Conference on Mass Spectrometry and Allied Topics, Chicago, IL, May, (2001)

47. Wu, C., Siems, W.F., Hill Jr., H.H.: Secondary electrospray ionization ion mobility spectrometry/mass spectrometry of illicit drugs. Anal. Chem. 72, 396-403 (2000)

48. de la Mora, J.F.: Ionization of vapor molecules by an electrospray cloud. Int. J. Mass Spectrom. 300, 182-193 (2011)

49. Wheeler, O.H.: The Girard reagents. J. Chem. Educ. 45, 435-437 (1968)

50. Sparrapan, R., Eberlin, L.S., Haddad, R., Cooks, R.G., Ebelin, M.N., Augusti, R.: Ambient Eberlin reactions via desorption electrospray ionization. J. Mass Spectrom. 41(9), 1242-1264 (2006)

51. Huang, G., Chen, H., Zhang, X., Cooks, R.G., Ouyang, Z.: Rapid screening of anabolic steroids in urine by reactive desorption electrospray ionization. Anal. Chem. 79(21), 8327-8332 (2007) 